\title{
Derivatization-free gel permeation chromatography elucidates enzymatic cellulose hydrolysis
}

Philip Engel ${ }^{1}$, Lea Hein ${ }^{1}$ and Antje C Spiess ${ }^{1,2^{*}}$

\begin{abstract}
Background: The analysis of cellulose molecular weight distributions by gel permeation chromatography (GPC) is a powerful tool to obtain detailed information on enzymatic cellulose hydrolysis, supporting the development of economically viable biorefinery processes. Unfortunately, due to work and time consuming sample preparation, the measurement of cellulose molecular weight distributions has a limited applicability until now.

Results: In this work we present a new method to analyze cellulose molecular weight distributions that does not require any prior cellulose swelling, activation, or derivatization. The cellulose samples were directly dissolved in dimethylformamide (DMF) containing 10-20\% (v/v) 1-ethyl-3-methylimidazolium acetate (EMIM Ac) for 60 minutes, thereby reducing the sample preparation time from several days to a few hours. The samples were filtrated $0.2 \mu \mathrm{m}$ to avoid column blocking, separated at $0.5 \mathrm{~mL} / \mathrm{min}$ using hydrophilic separation media and were detected using differential refractive index/multi angle laser light scattering (dRI/MALLS). The applicability of this method was evaluated for the three cellulose types Avicel, a-cellulose and Sigmacell. Afterwards, this method was used to measure the changes in molecular weight distributions during the enzymatic hydrolysis of the different untreated and ionic liquid pretreated cellulose substrates. The molecular weight distributions showed a stronger shift to smaller molecular weights during enzymatic hydrolysis using a commercial cellulase preparation for cellulose with lower crystallinity. This was even more pronounced for ionic liquid-pretreated cellulose.
\end{abstract}

Conclusions: In conclusion, this strongly simplified GPC method for cellulose molecular weight distribution allowed for the first time to demonstrate the influence of cellulose properties and pretreatment on the mode of enzymatic hydrolysis.

Keywords: Cellulose molecular weight, Gel permeation chromatography, Eluent for underivatized cellulose

\section{Background}

Gel permeation chromatography is a well-established technology to provide in-depth information on the cellulose polymer molecular weight distribution [1]. This can be particularly useful to understand and improve the enzymatic cellulose hydrolysis which is an essential aspect for efficient biomass utilization [2]. Until now cellulose pretreatment and enzymatic hydrolysis efficiency is primarily assessed by soluble sugar analysis [3,4] that is evaluated and correlated to the corresponding substrate

\footnotetext{
*Correspondence: antje.spiess@avt.rwth-aachen.de

'AVT-Enzyme Process Technology, RWTH Aachen University, Worringerweg 1, Aachen 52056, Germany

${ }^{2}$ Interactive Materials Research, DWI an der RWTH Aachen e.V, Forckenbeckstr. 50, Aachen 52074, Germany
}

properties of cellulose: crystallinity, particle size, and accessible surface area [5-7]. However, the focus on sugar formation excludes substantial aspects of the hydrolysis reaction: The enzymatic cellulose hydrolysis is performed by a mixture of different endo- and exo-acting enzymes. While exoglucanases cleave soluble cellobiose from the cellulose polymer, the endoglucanases cut the polymer in the interior of the chain, not necessarily resulting in direct soluble sugar formation [8]. The statistical release of shorter polymer products would provide information about hydrolysis and the effect of pretreatment efficiency or cellulose accessibility on the hydrolysis. Therefore, the investigation of enzymatic cellulose hydrolysis should focus on changes in the cellulose polymer during the reaction in addition to soluble sugar

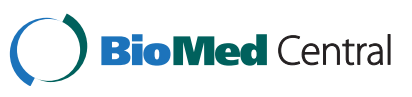


analysis and cellulose molecular weight distributions should be measured standard-wise to correlate them to cellulose types and pretreatment methods.

Despite the advantages of cellulose gel permeation chromatography to analyze cellulose molecular weight distributions during enzymatic hydrolysis, only very few studies performed GPC measurements due to a complex and labor intensive sample preparation $[9,10]$. The primary challenge for chromatographic analysis is the very low solubility of cellulose. An established method to dissolve cellulose is the prior derivatization with phenylisocyanate to form tricarbanilates for dissolution in tetrahydrofuran [11]. Other well established methods dissolve the cellulose in dimethylacetamide/LiCl or 1,3-dimethyl-2-imidazolidinone/LiCl (DMI/ $\mathrm{LiCl})[12,13]$. The latter methods have the advantage of analyzing non-derivatized cellulose but require swelling or activation of cellulose with ammonia or water before dissolution, or very long dissolution times $[14,15]$. As a result, the sample preparation for the analysis takes several days like for cellulose derivatization, significantly limiting the amount of samples that can be analyzed [16]. Therefore, new methods to prepare and analyze cellulose via GPC are highly desirable. One recent new approach has applied ionic liquids as solvent and eluent in cellulose analysis by GPC [17]. Ionic liquids are known to dissolve cellulose effectively [18]; but pose the disadvantage of high viscosity, requiring low flow rates of $0.01 \mathrm{~mL} / \mathrm{min}$ that lead to impractically long analysis times. In conclusion, a viable and robust method that can analyze cellulose quickly and without extensive sample preparation is still missing.

In this work we present a new GPC method that does not require any cellulose activation or derivatization prior to cellulose analysis. The cellulose dissolution is based on a recent report using organic solvents containing small amounts of ionic liquid [19]. Here, we used a mixture of DMF and EMIM Ac to dissolve cellulose and to elute cellulose from the GPC column. The new solvent system was evaluated for the three commonly used cellulose types Avicel, $\alpha$-cellulose and Sigmacell 101. As an application example, the effects of ionic liquid pretreatment on the cellulose molecular weight distributions during enzymatic hydrolysis were compared to that of native cellulose. Substantially altered cellulose molecular weight distributions during enzymatic hydrolysis suggest a different mode of action during enzymatic cellulose hydrolysis after ionic liquid pretreatment thus supporting the usefulness of cellulose molecular weight distribution analysis for biomass utilization.

\section{Results and discussion}

\section{GPC analysis of commercial cellulose preparations in DMF/EMIM AC}

To evaluate the new DMF/EMIM Ac solvent system for cellulose molecular weight distribution analysis by GPC, the three cellulose types Avicel, $\alpha$-cellulose and
Sigmacell were analyzed. MALLS measurement was used for absolute molecular weight determination because no ideal GPC standard for cellulose is available. In addition, MALLS measurement provides direct information on the quality of the separation method: A linear decrease of the logarithm of the molar mass with increasing elution volume indicates a pure size-based separation without stationary phase interaction. In Figure 1 the $d R I$ and molar mass signals for the three cellulose types and the resulting differential molecular weight distributions are given.

Figure 1a, c and e illustrate the quality of the measurement with this new GPC method. Based on the dRI signal the mass recovery was calculated to be $102 \%$, 99\% and $109 \%$ for Avicel, $\alpha$-cellulose and Sigmacell, respectively, indicating that cellulose was completely dissolved and detected in DMF/EMIM Ac with an acceptably low error. The dissolution of cellulose in DMF/EMIM Ac could be characterized further e.g. by nuclear magnetic resonance (NMR) or dynamic light scattering [20]. The mass recovery confirmed that the correct $d n / d c$ value of $0.168 \mathrm{~mL} / \mathrm{g}$ was used for this solvent system. The molecular weight signals of all three cellulose types showed a steadily declining molecular weight with increasing elution volume suggesting a truly size-based chromatographic separation. Beyond an elution volume of $30 \mathrm{~mL}$ lower molecular weight oligomers at relatively low concentrations eluted, resulting in increased noise of the light scattering signal due to the small refractive index increment. Nonetheless, all three cellulose types displayed the same slope of the log molar mass vs. elution volume regression, thereby indicating the high quality of the cellulose analysis. The differential molecular weight distributions were calculated using the corresponding parameters of the exponential fit lines.

From the differential molecular weight distributions in Figure $1 \mathrm{~b}, \mathrm{~d}$ and $\mathrm{f}$ the weight average molecular weights $M_{w}$ and the polydisperity, defined as $M_{w} / M_{n}$ (with $M_{n}$ being the number average molecular weight), of the distributions were determined (Table 1). This confirmed the expected average molecular weight ranges to be the smallest for Avicel and the largest for $\alpha$-cellulose. The measured $M_{w}$ values for the different cellulose types were in the same order of magnitude and sequence as previous results $[9,11,20-22]$. However, comparing $M_{w}$ values in the literature for the most defined cellulose, Avicel, shows variation between $0.49 \cdot 10^{4}$ and $4.9 \cdot 10^{4} \mathrm{~g} /$ mol $[9,11,22]$. This large variation can be attributed to a number of factors, i.e. lot-to-lot variability of the batches, different cellulose preparation methods, and most importantly different calibration standards used $[11,16,20]$. In particular, cellulose derivatization potentially results in a loss of lower molecular weight oligomers, leading to an overestimation of $M_{w}$ [9]. A possible 

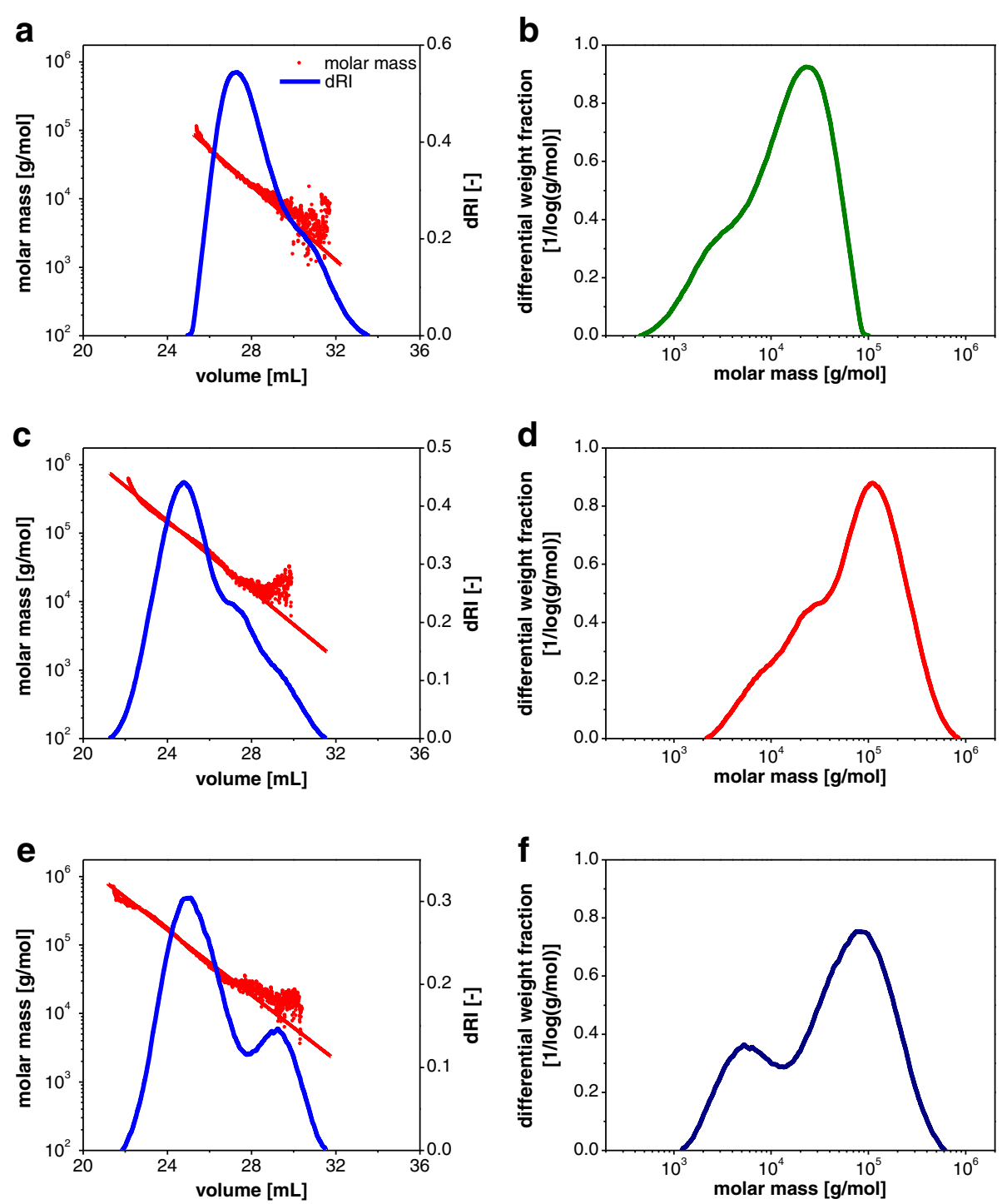

Figure 1 GPC measurement of different cellulose types. dRI and molecular mass elution profile for (a) Avicel, (c) a-cellulose, and (e) Sigmacell. Resulting differential weight distributions calculated for (b) Avicel, (d) a -cellulose and (f) Sigmacell. Eluent DMF/10\% (v/v) EMIM Ac, $100 \mu \mathrm{L}$ injection with $1 \mathrm{~g} / \mathrm{L}$ cellulose and flow rate $0.5 \mathrm{~mL} / \mathrm{min}$.

derivatization of the reducing end of cellulose or degradation by EMIM Ac has been reported [23-25]. In this study, the cellulose degradation was minimized by applying moderate temperatures of $<80^{\circ} \mathrm{C}$ and short incubation times of $1 \mathrm{~h}$ for dissolution. A potential derivatization of cellulose at the reducing end would not

Table 1 Cellulose substrate characteristics

\begin{tabular}{llll}
\hline & $\boldsymbol{M}_{\boldsymbol{w}} \mathbf{g} \mathbf{g} \mathbf{m o l}$ & Polydispersity, - & $\mathbf{C r l}, \mathbf{~}$ \\
\hline Avicel PH 101 & 28,400 & 3.1 & $82 \%$ \\
Sigmacell 101 & 76,100 & 4.7 & nd \\
a-cellulose & 109,000 & 3 & $64 \%$ \\
\hline
\end{tabular}

Weight-average molecular weight and polydispersity was estimated from differential weight distributions shown in Figure $1 \mathrm{~b}, \mathrm{~d}$, f. Crystallinity indices $\mathrm{Crl}$ are from Jäger et al. [20,26]. significantly alter GPC analysis because the derivatized reducing end causes only negligible changes of the molecular weight of the high molecular weight polymer.

\section{Comparison of untreated and ionic liquid-pretreated and enzymatically hydrolysed commercial celluloses using GPC}

After showing the general applicability of the new GPC method for cellulose analysis, the changes in the molecular weight distribution during enzymatic cellulose hydrolysis were investigated to understand how different cellulose properties and ionic liquid (IL) pretreatment affect the mechanism of the enzymatic hydrolysis. The differential molecular weight distributions during the 
hydrolysis of native and regenerated Avicel, $\alpha$-cellulose and Sigmacell are compared in Figure 2.

The areas under the curves in Figure 2 correspond to the sugar based conversion of cellulose measured by high performance liquid chromatography (HPLC) and provided in the figure legend. During hydrolysis of untreated Avicel in Figure 2a the height of the distribution reduced along with the conversion. No significant change in the relative molecular weight distribution was observable up to the conversion of 33\% reached after $48 \mathrm{~h}$. Obviously, soluble sugars were produced almost exclusively from the cellulose polymer without affecting the relative molecular weight distribution. This result can be attributed to the structural properties of Avicel, which is microcrystalline cellulose with a crystallinity of $82 \%$ and a well-defined particle size of $44 \mu \mathrm{m}$ on average $[27,28]$. Crystalline cellulose with a low accessible surface area is primarily hydrolyzed by exoglucanases that form cellobiose directly from cellulose $[8,26,29]$. Endoglucanases require a higher accessibility of the polymer. They hydrolyze only amorphous regions of cellulose in the interior of the polymer, resulting in reduced
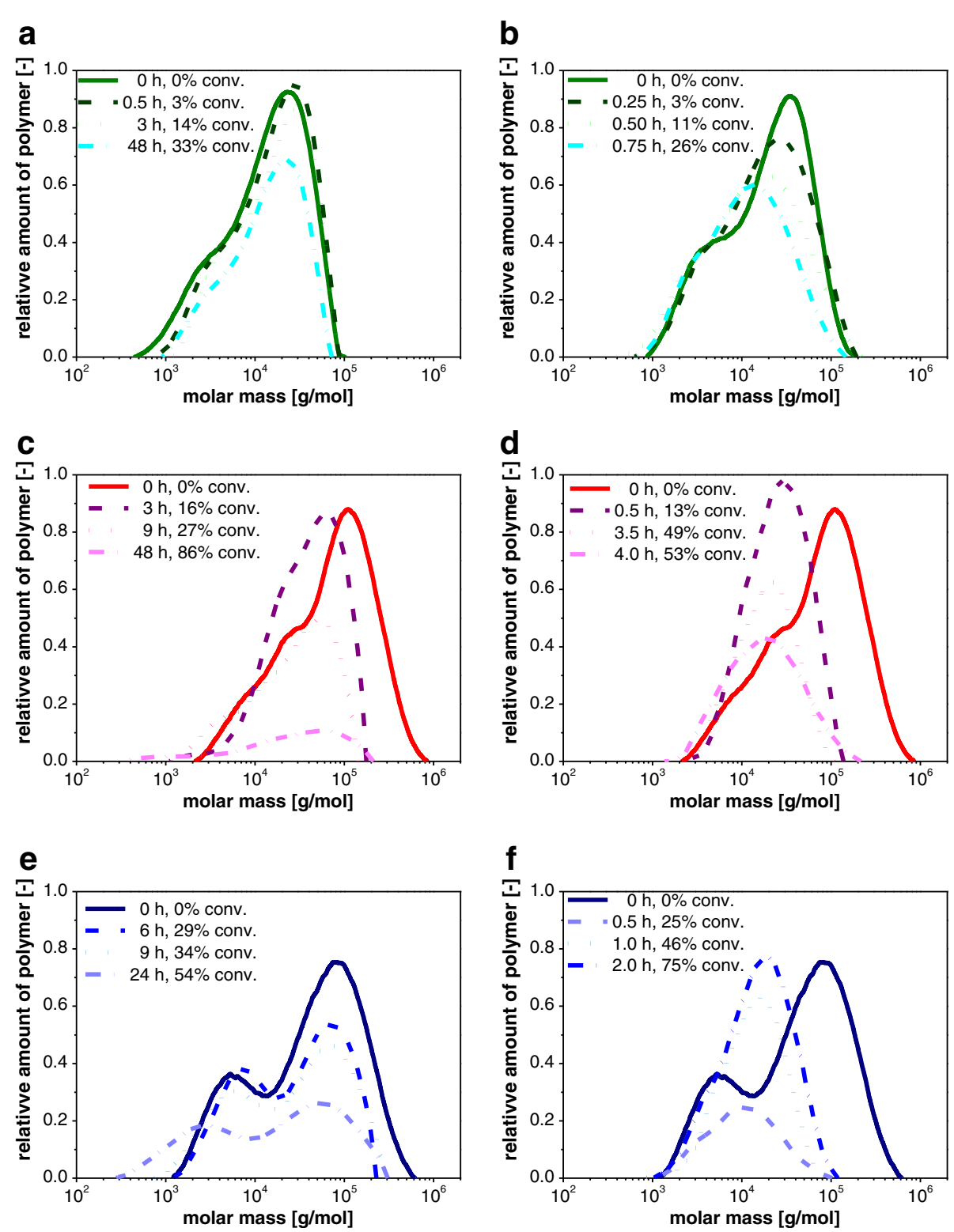

Figure $\mathbf{2}$ Changes in differential weight distributions at different times during enzymatic hydrolysis. (a, c, e) untreated and (b, d, f) regenerated cellulose using $(\mathbf{a}, \mathbf{b})$ Avicel, $(\mathbf{c}, \mathbf{d}) \mathbf{a}$-cellulose $(\mathbf{e}, \mathbf{f})$ Sigmacell. Areas under the curves were adjusted to represent weight loss during the hydrolysis. Enzymatic hydrolysis performed at $45^{\circ} \mathrm{C}$ using $0.25 \mathrm{~g} / \mathrm{L}$ desalted Celluclast ${ }^{\circledR}$ in $0.1 \mathrm{M} \mathrm{NaAc}$ buffer pH 4.8. Eluent DMF/10\% (v/v) EMIM Ac, flow rate $0.5 \mathrm{~mL} / \mathrm{min}$. 
molecular weights [26]. Therefore, the results in Figure $2 \mathrm{a}$ demonstrate that endoglucanases play a minor role in the hydrolysis of untreated Avicel explaining the negligible shift in the molecular weight distributions at low conversion levels.

The first important observation in Figure $2 b, d$ and $f$ was that the hydrolysis reaction of regenerated cellulose proceeded drastically faster, which is in accordance with earlier results [30]. Furthermore, the ionic liquid pretreatment as it was performed here did not change the initial molecular weight distributions of the cellulose before the hydrolysis.

Other than the hydrolysis of untreated Avicel, the hydrolysis of Avicel regenerated from ionic liquids in Figure $2 b$ resulted in a shift of the molecular weight distributions to lower molecular weights and a reduction of the $M_{w}$ by $37 \%$ at a conversion of only $26 \%$. Additionally, the height of the distribution reduced with increasing conversion, similar to that of untreated Avicel (Figure 2a). Differences in the hydrolysis of untreated and regenerated Avicel with the same cellulase mixture could be linked to the properties of regenerated cellulose: Regenerated cellulose is highly porous and amorphous, giving access to the individual cellulose polymer chains for the cellulases and also providing cleavage sites for endoglucanase [31]. Consequently, cellulose was now hydrolyzed in a synergistic manner, not only reducing the distributions in height but also shifting them to lower molecular weights. In summary, the cellulose pretreatment caused a shift of the hydrolysis mechanism from a merely exoglucanase activity to a combined exo- and endo-activity, resulting in stronger changes in the differential molecular weight distribution and additionally in a much faster conversion.

The enzymatic hydrolysis of untreated $\alpha$-cellulose and Sigmacell is shown in Figure 2c and e, respectively. In contrast to untreated Avicel, a shift of the molecular weight distributions to lower molecular weights during enzymatic hydrolysis was observed for untreated $\alpha$-cellulose and Sigmacell. In particular Sigmacell showed the strongest reduction in $M_{w}$ by $43 \%$ from $7.61 \cdot 10^{4}$ to $4.36 \cdot 10^{4} \mathrm{~g} / \mathrm{mol}$ at $54 \%$ conversion. Again, these results can be attributed to the structural properties of the two different cellulose types. Both were more amorphous than Avicel with a crystallinity of $64 \%$ for $\alpha$-cellulose and no detectable crystallinity for Sigmacell $[27,30]$. Consequently, untreated $\alpha$-cellulose and Sigmacell was more accessible to endo- and exoglucanases resulting in shifting differential molecular weight distributions. Nonetheless, even at high conversion there were still significant amounts of high molecular weight polymers remaining. E.g. after $48 \mathrm{~h} \alpha$-cellulose was converted to $86 \%$, but contained still polymers larger than $2 \cdot 10^{5} \mathrm{~g} / \mathrm{mol}$. Probably these were nonhydrolysable crystalline cellulose residuals.

The hydrolysis of regenerated $\alpha$-cellulose and Sigmacell is shown in Figure 2d and f, respectively. Same as for regenerated Avicel, both substrates are amorphous, resulting in a shift of the differential molecular weight distributions to lower molecular weight during hydrolysis. For both substrates the $M_{w}$ of the distribution strongly declined even at very low conversion: The $M_{w}$ of regenerated $\alpha$-cellulose reduced by $43 \%$ at less than $2 \%$ conversion. This was probably due to enhanced initial endoglucanase activity on the highly accessible regenerated cellulose and hints at a challenge in measuring the changes in the molecular weight distributions with high resolution at early incubation times: The hydrolysis reaction has to be quickly quenched to successfully 'freeze' the reaction progress. Otherwise, residual enzymatic activity will lead to further hydrolysis of the pre-treated substrate until analysis. In particular for pretreated substrates where endo-attack of long polymer chains can easily occur, this leads to errors in the estimation of the $M_{w}$.

In addition to the reduction in $M_{w}$, also the shape of the molecular weight distribution changed during enzymatic hydrolysis (Figure 2). In particular, the differential molecular weight distributions of regenerated Sigmacell and $\alpha$-cellulose and became narrower during hydrolysis. To illustrate the change of the distribution, the polydispersity during hydrolysis is depicted in Figure 3 for all untreated and regenerated celluloses.

The polydispersity of untreated cellulose increased substantially with increasing conversion except for Avicel that only reached very low conversion levels. An increasing polydispersity implies that the distribution becomes wider during hydrolysis: The number of smaller molecular weight polymers increased while the high molecular weight polymers were still present. In contrast to untreated cellulose, the polydispersity of regenerated $\alpha$-cellulose and Sigmacell gradually declined with increasing conversion which is in accordance with the degradation of the high molecular weight polymers.

Previous studies that have investigated cellulose molecular weight distributions during hydrolysis include enzymatic hydrolysis [9], chemical hydrolysis [32-34] or both [10]. In summary it was observed that the cellulose molecular weight distributions changed much stronger for chemical hydrolysis than for enzymatic hydrolysis, which showed good agreement to our results. Chemical hydrolysis cleaves cellulose chains statistically reducing the polymer length at early reaction times. In contrast, cellulase mixtures are naturally evolved to convert cellulose effectively and selectively to soluble sugars for microbial uptake. The present study compared different cellulose types and the effect of ionic liquid pretreatment on enzymatic hydrolysis for the first time. In future, the combination of sugar measurements and cellulose polymer molecular weight distributions will contribute fundamentally to understand enzymatic cellulose hydrolysis, to evaluate pretreatment strategies, and to design optimal cellulase mixtures. 


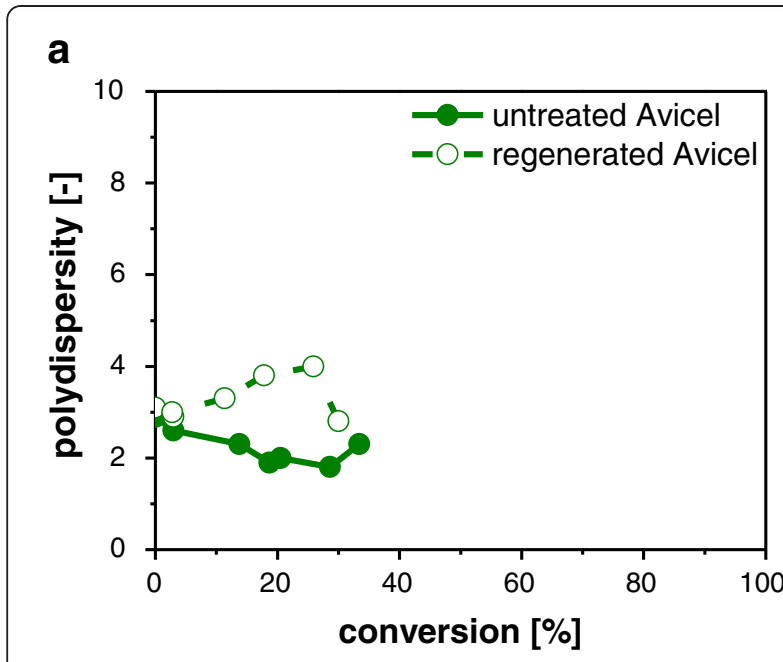

b

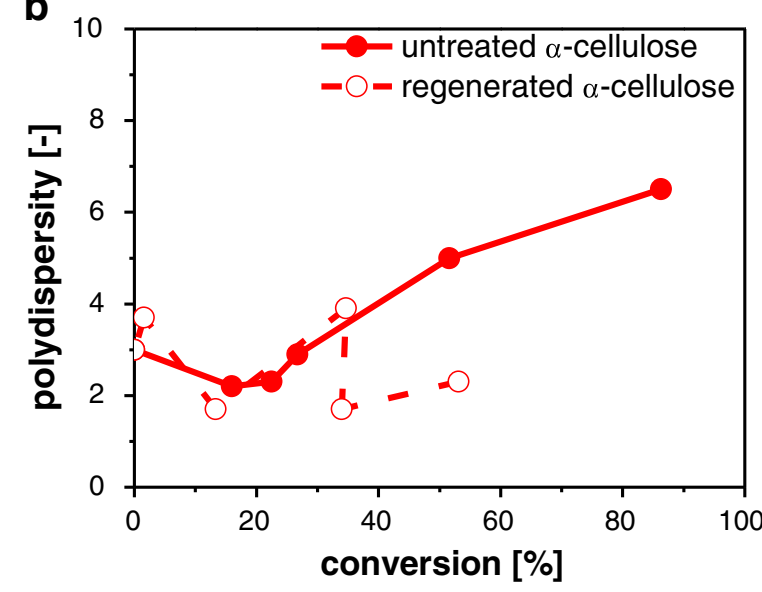

$$
\text { C }
$$

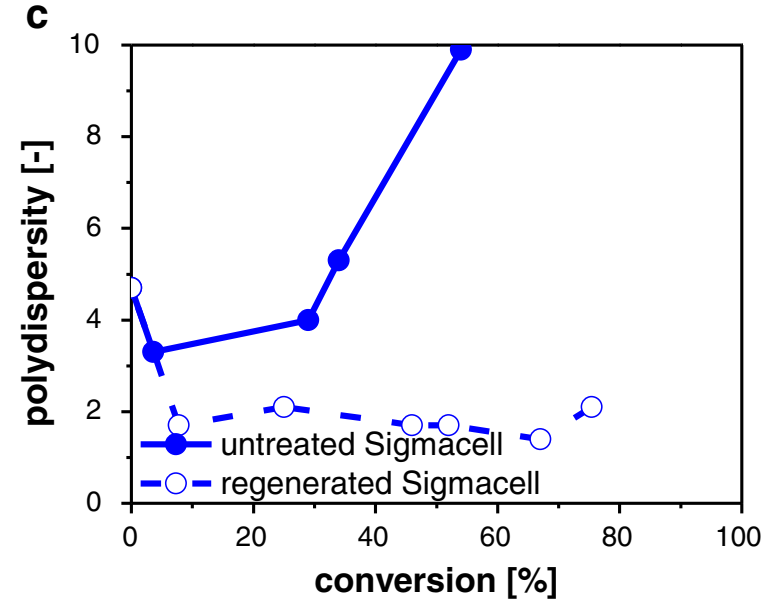

Figure 3 Changes in polydispersity during enzymatic hydrolysis of untreated and regenerated cellulose. (a) Avicel, (b) Sigmacell and (c) a-cellulose. Enzymatic hydrolysis performed at $45^{\circ} \mathrm{C}$ using $0.25 \mathrm{~g} / \mathrm{L}$ desalted Celluclast ${ }^{\circledR}$ in $0.1 \mathrm{M} \mathrm{NaAc}$ buffer $\mathrm{pH}$ 4.8. Eluent DMF/10\% (v/v) EMIM Ac, flow rate $0.5 \mathrm{~mL} / \mathrm{min}$.

\section{Conclusions}

We presented a new chromatographic method for cellulose GPC analysis employing DMF/EMIM Ac as cellulose solvent and eluent that eliminates time intensive sample preparation and allows to measure larger sample numbers necessary for in-depth understanding of enzymatic cellulose hydrolysis. This method can potentially be extended for the measurement of lignin and wood, similar to earlier approaches working with ionic liquids $[21,35]$. If the analysis of the cellulose molecular weight distributions will be extended from the pure qualitative evaluation performed in this study to a quantitative analysis and interpretation using mechanistically sound mathematical models of enzymatic cellulose degradation, this novel GPC analysis will be able to play a key role in building a holistic understanding of enzymatic cellulose hydrolysis.

\section{Methods}

\section{Materials}

The cellulose substrates Avicel PH101, Sigmacell 101, and $\alpha$-cellulose were purchased from Sigma-Aldrich (St Louis, USA). The ionic liquid 1-ethyl-3-methylimidazolium acetate (EMIM Ac) was kindly provided by BASF (Ludwigshafen, Germany). The commercial cellulase preparation Celluclast ${ }^{\circledR} 1.5 \mathrm{~L}$ from T. reesei ATCC26621 (Novozyme, Denmark) with an activity of 700 EGU/g according to manufacture specification was used in the hydrolysis experiments. Prior to cellulose hydrolysis, the cellulase was rebuffered in $0.1 \mathrm{M}$ sodium acetate buffer pH 4.8 with a HiPrep 26/10 desalting column (GE Healthcare, Buckinghamshire, Great Britain) using an ÄKTA FPLC system (GE Healthcare) [28]. The rebuffered Celluclast ${ }^{\circledR}$ had a specific activity of $244 \mathrm{U} / \mathrm{g}$ according to the standard filter paper assay $[26,36]$.

\section{Sample preparation}

Dry cellulose was dissolved for the GPC measurement without any prior swelling, activation, or derivatization. 1-2 $\mathrm{g} / \mathrm{L}$ cellulose was dissolved in dimethylformamide (DMF) containing $10-20 \%(\mathrm{v} / \mathrm{v})$ EMIM Ac at $80^{\circ} \mathrm{C}$ for 1 h. 2 g/L Avicel dissolved almost instantaneously in DMF containing 10\% (v/v) EMIM Ac and formed a visually clear solution. $1 \mathrm{~g} / \mathrm{L} \alpha$-cellulose and Sigmacell 101 in DMF containing 20\% (v/v) EMIM Ac formed a clear solution within less than 10 minutes. The volume percentage EMIM Ac required for dissolution should generally increase with degree of polymerisation and concentration of cellulose to be dissolved. For measurement of cellulose during enzymatic hydrolysis, $1 \mathrm{~mL}$ samples, containing $10 \mathrm{mg}$ cellulose were dried over night at $80^{\circ} \mathrm{C}$ prior to the dissolution of $1 \mathrm{mg}$ dried cellulose in $1 \mathrm{~mL}$ DMF/EMIM Ac. Before injection, the 
samples were filtered with a Whatman $1 \mu \mathrm{m}$ polytetrafluorethylene (PTFE) filter (GE Healthcare).

\section{Gel permeation chromatography}

The gel permeation chromatography system consisted of an isocratic pump, auto-sampler with thermostat (Agilent 1200 series, Santa Clara, USA); and a set of GRAM separation columns, one $30 \AA$ and two $10000 \AA$ columns (PSS, Mainz, Germany) kept at $60^{\circ} \mathrm{C}$ in a column oven (K7, Techlab, Erkerode, Germany). A MALLS detector was used for absolute molecular weight measurement (DAWN HELEOS 8+ $\lambda 658 \mathrm{~nm}$, Wyatt Technologies, Santa Barbara, USA) and a differential refractive index (dRI) detector (Optilab rEX $\lambda 658 \mathrm{~nm}$, Wyatt Technologies) for quantification. Data acquisition of molecular weight was performed with Astra Software (Wyatt Technologies) using Zimm first order as model. $100 \mu \mathrm{L}$ samples with $1-2 \mathrm{~g} / \mathrm{L}$ cellulose in DMF containing $10-20 \%(\mathrm{v} / \mathrm{v})$ EMIM Ac were injected and analyzed at a flow rate of $0.5 \mathrm{~mL} / \mathrm{min}$ DMF containing $10 \%(\mathrm{v} / \mathrm{v})$ EMIM Ac as eluent. Linear regression of $\log$ MW vs. retention time resulted in $\mathrm{R}^{2}$ larger than 0.9 for all samples. Replicate samples resulted in deviations of $<10 \%$ with respect to $M_{W}$ and $M_{n}$, respectively.

\section{Measurement of specific refractive index increment}

The refractive index increment $(d n / d c)$ for cellulose in DMF/10\% (v/v) EMIM Ac was determined using Optilab rEX dRI detector. Ten 20-100 $\mu \mathrm{L}$ samples of $0.5 \mathrm{~g} / \mathrm{L}$ cellulose in DMF/10\% (v/v) EMIM Ac were directly injected into the dRI detector. From the peak in the dRI detector the $d n / d c$ value was then calculated based on $100 \%$ mass recovery in the ASTRA software. The resulting $d n / d c$ value was $0.168 \pm 0.006 \mathrm{~mL} / \mathrm{g}$. This value was further confirmed by the GPC measurements of the cellulose, showing a mass recovery in the dRI signal of 95$110 \%$ in all measurements.

\section{Cellulose pretreatment}

The regenerated cellulose from ionic liquid pretreatment was obtained by dissolving $50 \mathrm{~g} / \mathrm{L}$ of the respective cellulose in EMIM Ac at $80^{\circ} \mathrm{C}$ in a thermo mixer MHR23 (HLC Biotech, Bovenden, Germany) for $1 \mathrm{~h}$ until a clear solution was formed. The cellulose was precipitated by $0.1 \mathrm{M}$ sodium acetate buffer $\mathrm{pH} 4.8$ while vigorously agitating. The regenerated cellulose was washed subsequently with 100 fold buffer volume to remove residual ionic liquid. The regenerated cellulose was kept wet at $4-8^{\circ} \mathrm{C}$ until use. The dry mass fraction of the regenerated cellulose was obtained by drying a cellulose sample at $104^{\circ} \mathrm{C}$ over night. The dry mass fraction of the different cellulose types were determined to $8.3 \% 10.4 \%$ and $9 \%$ for Avicel, $\alpha$-cellulose, and Sigmacell, respectively.

\section{Enzymatic hydrolysis}

Cellulose hydrolysis was performed at $45^{\circ} \mathrm{C}$ in $0.1 \mathrm{M}$ sodium acetate buffer $\mathrm{pH} 4.8$ with $10 \mathrm{~g} / \mathrm{L}$ cellulose and $0.25 \mathrm{~g} / \mathrm{L}$ desalted cellulase in $15 \mathrm{~mL}$ Falcon tubes in a MHR 23 (HLC Biotech, Bovenden, Germany). To stop the enzymatic hydrolysis, the samples were transferred to an ice bath for $20 \mathrm{~min}$. The undissolved cellulose was afterwards removed by centrifugation at $4^{\circ} \mathrm{C}$. The glucose and cellobiose concentration in the supernatant were determined by Dionex HPLC with refractive index detector (STH 585, $232 \mathrm{XL}$, E580) using an 300x4 mm organic acid column (CS-Chromatographie, Langerwehe, Germany) with a flow rate of $1 \mathrm{~mL} / \mathrm{min}$ and $5 \mathrm{mM}$ $\mathrm{H}_{2} \mathrm{SO}_{4}$ as eluent. The undissolved cellulose pellet was further inactivated at $100^{\circ} \mathrm{C}$ in a water bath for $10 \mathrm{~min}$ and afterwards dried at $85^{\circ} \mathrm{C}$ over night before the GPC measurement.

\section{Abbreviations}

DMF: DiMethylFormamide; dRI: Differential refractive index; EMIM Ac: 1-Ethyl3-Methyl-Imidazolium acetate; GPC: Gel permeation chromatography; HPLC: High performance liquid chromatography; IL: Ionic liquid; MALLS: Multi angle laser light scattering; NMR: Nuclear magnetic resonance;

PTFE: PolyTetraFluorEthylene.

\section{Competing interests}

The authors declare no competing interests.

\section{Authors' contributions}

PE designed the experiments, LH and PE performed the cellulose sample preparations and analyses as well as the enzymatic reactions. PE analysed the data and drafted the manuscript. AS conceived the study and helped to draft the manuscript. All authors read and approved the final manuscript.

\section{Acknowledgements}

This work was performed as part of the Cluster of Excellence 236 "TailorMade Fuels from Biomass", which is funded by the excellence initiative by the German federal and state governments to promote science and research at German universities. Additional support by the Deutsche

Forschungsgemeinschaft (German Research Foundation) within research training group 1166 "Biocatalysis using Non-Conventional Media -BioNoCo" is gratefully acknowledged. The authors thank BASF for kindly providing the ionic liquid used in this study. Furthermore the authors thank Benjamin Bonhage for the fruitful discussions.

Received: 16 August 2012 Accepted: 5 October 2012

Published: 12 October 2012

\section{References}

1. Eremeeva T: Size-exclusion chromatography of enzymatically treated cellulose and related polysaccharides: a review. J Biochem Biophys Methods 2003, 56(1-3):253-264.

2. Lynd LR, Laser MS, Brandsby D, Dale BE, Davison B, Hamilton R, Himmel M, Keller M, McMillan JD, Sheehan J, Wyman CE: How biotech can transform biofuels. Nat Biotechnol 2008, 26(2):169-172.

3. Lynd LR, Weimer PJ, van ZyI WH, Pretorius IS: Microbial cellulose utilization: Fundamentals and biotechnology. Microbiol Mol Biol R 2002, 66(3):506-577.

4. Zhang YHP, Himmel ME, Mielenz JR: Outlook for cellulase improvement: Screening and selection strategies. Biotechnol Adv 2006, 24(5):452-481.

5. Arantes V, Saddler JN: Cellulose accessibility limits the effectiveness of minimum cellulase loading on the efficient hydrolysis of pretreated lignocellulosic substrates. Biotechnol Biofuels 2011, 4:3.

6. Arantes V, Saddler JN: Access to cellulose limits the efficiency of enzymatic hydrolysis: the role of amorphogenesis. Biotechnol Biofuels 2010, 3:4. 
7. Rollin JA, Zhu Z, Sathitsuksanoh N, Zhang YHP: Increasing cellulose accessibility is more important than removing lignin: a comparison of cellulose solvent-based lignocellulose fractionation and soaking in aqueous ammonia. Biotechnol Bioeng 2011, 108(1):22-30.

8. Mosier NS, Hall P, Ladisch CM, Ladisch MR: Reaction kinetics, molecular action, and mechanisms of cellulolytic proteins. Adv Biochem Eng Biotechnol 1999, 65:23-40.

9. Pala H, Mota M, Gama FM: Enzymatic depolymerisation of cellulose. Carbohyd Polym 2007, 68(1):101-108.

10. Strobin G, Ciechanska D, Wawro D, Boryniec S, Struszczyk H, Sobczak S: Gel permeation chromatography of chemically, irradiated and enzymatically treated cellulosics. Fibres Text East Eur 2003, 11(4):62-65.

11. Evans R, Wearne RH, Wallis AFA: Molecular-weight distribution of cellulose as its tricarbanilate by high-performance size exclusion chromatography. J Appl Polym Sci 1989, 37(12):3291-3303.

12. Dawsey TR, McCormick CL: The lithium chloride/dimethylacetamide solvent for cellulose - a literature-review. J Macromol Sci R M C 1990 C30(3-4):405-440.

13. Yanagisawa M, Isogai A: SEC-MALS-QELS study on the molecular conformation of cellulose in $\mathrm{LiCl} /$ amide solutions. Biomacromolecules 2005, 6(3):1258-126.

14. Rosenau T, Potthast A, Adorjan I, Hofinger A, Sixta H, Firgo H, Kosma P: Cellulose solutions in N-methylmorpholine-N-oxide (NMMO) degradation processes and stabilizers. Cellulose 2002, 9(3-4):283-291.

15. Striegel AM, Timpa JD: Molecular characterization of polysaccharides dissolved in $\mathrm{Me}_{2} \mathrm{NAC} \mathrm{LiCl}$ by gel permeation chromatography. Carbohydr Res 1995, 267:271-290.

16. Henniges U, Kostic M, Borgards A, Rosenau T, Potthast A: Dissolution behavior of different celluloses. Biomacromolecules 2011, 12(4):871-879.

17. Fukaya Y, Tsukamoto A, Kuroda K, Ohno H: High performance "ionic liquid" chromatography. Chem Commun 2011, 47(7):1994-1996.

18. Swatloski RP, Spear SK, Holbrey JD, Rogers RD: Dissolution of cellose with ionic liquids. J Am Chem Soc 2002, 124(18):4974-4975.

19. Rinaldi R: Instantaneous dissolution of cellulose in organic electrolyte solutions. Chem Commun 2011, 47(1):511-513.

20. Röder T, Morgenstern B, Schelosky N, Glatter O: Solutions of cellulose in N, $\mathrm{N}$-dimethylacetamide/lithium chloride studied by light scattering methods. Polymer 2001, 42(16):6765-6773.

21. Guerra A, Mendonca R, Ferraz A: Molecular weight distribution of wood components extracted from Pinus taeda biotreated by Ceriporiopsis subvermispora. Enzyme Microb Tech 2003, 33(1):12-18.

22. Schelosky N, Roder T, Baldinger T: Molecular mass distribution of cellulosic products by size exclusion chromatography in DMAc/LiCl. Papier 1999, 53(12):728-738.

23. Pinkert A, Marsh KN, Pang SS: Reflections on the solubility of cellulose. Ind Eng Chem Res 2010, 49(22):11121-11130.

24. Pinkert A, Marsh KN, Pang SS, Staiger MP: lonic liquids and their interaction with cellulose. Chem Rev 2009, 109(12):6712-6728.

25. Ebner $G$, Hofinger A, Brecker $L$, Rosenau T: Commentary on the alleged "irregularities" in APT spectra of imidazolium-based ionic liquids. Cellulose 2008, 15(6):763-767.

26. Zhang YHP, Lynd LR: Toward an aggregated understanding of enzymatic hydrolysis of cellulose: Noncomplexed cellulase systems. Biotechnol Bioeng 2004, 88(7):797-824.

27. Jäger G, Wu Z, Garschhammer K, Engel P, Klement T, Rinaldi R, Spiess AC, Büchs J: Practical screening of purified cellobiohydrolases and endoglucanases with a-cellulose and specification of hydrodynamics. Biotechnol Biofuels 2010, 3:18.

28. Engel $P$, Mladenov R, Wulfhorst $H$, Jäger $G$, Spiess AC: Point by point analysis: how ionic liquid affects the enzymatic hydrolysis of native and modified cellulose. Green Chem 2010, 12(11):1959-1966.

29. Hall M, Bansal P, Lee JH, Realff MJ, Bommarius AS: Cellulose crystallinity - a key predictor of the enzymatic hydrolysis rate. FEBS J 2010, 277(6):1571-1582.

30. Jäger G, Wulfhorst H, Zeithammel EU, Elinidou E, Spiess AC, Büchs J: Screening of cellulases for biofuel production: online monitoring of the enzymatic hydrolysis of insoluble cellulose using high-throughput scattered light detection. Biotechnol J 2011, 6(1):74-85.

31. Zhao H, Jones CIL, Baker GA, Xia S, Olubajo O, Person VN: Regenerating cellulose from ionic liquids for an accelerated enzymatic hydrolysis. J Biotechnol 2009, 139(1):47-54.
32. Rinaldi P, Schüth F: Depolymerization of cellulose using solid catalysts in ionic liquids. Angew Chem Int Ed 2008, 47(42):8047-8050.

33. Rinaldi R, Meine N, vom Stein J, Palkovits R, Schüth F: Which controls the depolymerization of cellulose in ionic liquids: The solid acid catalyst or cellulose? ChemSusChem 2010, 3(2):266-276

34. Isogai T, Yanagisawa M, Isogai A: Degrees of polymerization (DP) and DP distribution of dilute acid-hydrolyzed products of alkali-treated native and regenerated celluloses. Cellulose 2008, 15(6):815-823.

35. Zoia L, King AWT, Argyropoulos DS: Molecular weight distributions and linkages in lignocellulosic materials derivatized from ionic liquid media. J Agric Food Chem 2011, 59(3):829-838.

36. Ghose TK: Measurement of cellulase activities. Appl Chem 1987, 59(2):257-268.

doi:10.1186/1754-6834-5-77

Cite this article as: Engel et al:: Derivatization-free gel permeation chromatography elucidates enzymatic cellulose hydrolysis. Biotechnology for Biofuels 2012 5:77.

\section{Submit your next manuscript to BioMed Central and take full advantage of:}

- Convenient online submission

- Thorough peer review

- No space constraints or color figure charges

- Immediate publication on acceptance

- Inclusion in PubMed, CAS, Scopus and Google Scholar

- Research which is freely available for redistribution

Submit your manuscript at www.biomedcentral.com/submit
C BioMed Central 